\title{
Optimized Model of Experimental Teaching Plan Based on Improved by Clear Theory
}

\author{
Xia Xinfeng ${ }^{1 *}$, Lv Weiwei $^{1}$ \\ ${ }^{1}$ (School of Mechanical and Vehicle Engineering, Beijing Institute of Technology, Beijing, Postcode: 100081)
}

\begin{abstract}
With the continuous advancement of the "Double First-Class" university plan, the status and role of experimental teaching in university teaching has become increasingly prominent. Chosen as one of the "Double First-Class" universities, Beijing Institute of Technology has also carried out a series of experimental teaching reforms, and has produced different reform plans in the laboratory of "Geometrical Accuracy Specifications", School of Mechanical Engineering. In order to optimize different schemes, the experimental teaching center innovatively proposes an optimized model improved by clear theory. This is a decisionmaking method based on triangular fuzzy number and clear theory. Utilizing the fuzzy theory and clear theory, the advantages and disadvantages of the alternatives can be ranked more accurately, the influence of subjective and objective factors in the process of selecting the alternatives can be reduced, and new methods can be provided for decision makers to choose the best alternative.
\end{abstract}

\section{Introduction}

The construction of "double first-class" is the focus of current university builders, and its proposal especially creates new opportunities for the development of local universities in our country. With the continuous advancement of the "double first-class" construction, the status and role of experimental teaching in college teaching have become increasingly prominent [1]. Experimental teaching is an important part of the teaching work of colleges and universities, and strengthening experimental teaching is an important means to cultivate students' innovative consciousness, innovative spirit and innovative ability [2, 3]. However, due to historical reasons, experimental teaching has always been in the auxiliary position of higher education and has not received enough attention. As a result, the content and form are outdated, and they have not kept up with the development of higher education and the times, and cannot meet the actual needs of modern talent training. Therefore, it is necessary to promote experimental teaching reform.

As a "double first-class" university, Beijing Institute of Technology has also carried out a series of experimental teaching reforms, and different reform plans have been produced in the Geometric Precision Standards Laboratory of the School of Machinery and Vehicles. In order to optimize the different programs, this paper establishes a clear theoretically improved experimental teaching program optimization model.

The publication of the book "Clarity Sets and Their Applications" [4] compiled by Wu Huaying and Professor $\mathrm{Wu}$ Heqin marked the birth of a new mathematical theory-the Clarity Set Theory. Professor Su Fahui collected the latest research results of Clarity Collection for many years, edited and integrated it, and made it systematized, and published the book "The Theoretical Basis of Clarity" [5]. It is more effective, more accurate, and more solid theoretical foundation than other theories when dealing with fuzzy phenomena that are partly partly part of it. This is a breakthrough and innovation in the research of expressing and processing fuzzy information.

The existing scheme decision model research is mostly fuzzy decision model $[6,7,8,9,10,11,12]$, but due to many shortcomings of fuzzy theory, the use of this decision model has certain restrictions $[13,14,15]$. This paper introduces the triangular fuzzy number theory in the fuzzy number theory on the basis of the clear theory [16, 17, 18], and establishes the experimental teaching program optimization model for the improvement of the clear theory. Triangular fuzzification of the evaluation value reduces the deviation caused by personal preference or statistical error in the judgment process, and the use of clear theory for evaluation and feedback in decisionmaking makes up for the defects of fuzzy theory. This makes the optimization result more accurate, provides a more reliable selection model for the optimization of a variety of experimental teaching programs, and is applied in the optimization process of the experimental teaching program of "Geometric Precision Norm", and has achieved good experimental results.

* (Email: xiafengfeng1989@163.com) 


\section{Basic principles}

\subsection{The definition of triangular fuzzy numbers and related operations}

\subsubsection{Definition of triangular fuzzy number}

Definition 1 [5] If $\mathbf{a}=\left[a^{L}, a^{M}, a^{U}\right]$, among them $0<a^{L}<a^{M}<a^{U}$ then it is said that $\mathrm{a}$ is a triangula $a^{L}, a^{M}, a^{U}$ r fuzzy number, the supremum, median and infimum of the fuzzy number are respectively $a^{L}, a^{M}, a^{U}$, call $a^{U}-a^{L}$ the value interval of the triangular fuzzy number.

\subsubsection{Related operations of triangular fuzzy numbers}

Definition 2[5, 6] Suppose two triangular fuzzy numbers $\mathbf{a}=\left[a^{L}, a^{M}, a^{U}\right], \mathbf{b}=\left[\mathrm{b}^{L}, b^{M}, b^{U}\right], \quad \mathrm{R}$ is the set of real numbers, where $0<a^{L}<a^{M}<a^{U}, 0<b^{L}<b^{M}<b^{U}$, then:

Addition operation:

$\mathbf{a}+\mathbf{b}=\left[\mathrm{a}^{\mathrm{L}}+\mathrm{b}^{\mathrm{L}}, \mathrm{a}^{\mathrm{M}}+\mathrm{b}^{\mathrm{M}}, \mathrm{a}^{\mathrm{U}}+\mathrm{b}^{\mathrm{U}}\right]$

Multiplication:

$\mathbf{a b}=\left[\mathrm{a}^{\mathrm{L}} \mathrm{b}^{\mathrm{L}}, \mathrm{a}^{\mathrm{M}} \mathrm{b}^{\mathrm{M}}, \mathrm{a}^{\mathrm{U}} \mathrm{b}^{\mathrm{U}}\right]$

Division operation:

$\mathbf{a} / \mathbf{b}=\left[\mathrm{a}^{\mathrm{L}} / \mathrm{b}^{\mathrm{L}}, \mathrm{a}^{\mathrm{M}} / \mathrm{b}^{\mathrm{M}}, \mathrm{a}^{\mathrm{U}} / \mathrm{b}^{\mathrm{U}}\right]$

Multiplication:

$\lambda \mathbf{a}=\left[\lambda a^{L}, \lambda a^{M}, \lambda a^{U}\right]$

\subsection{Clear theoretical definitions and related operations}

\subsubsection{Definition of clear rational numbers}

Definition 3: Set the domain $U=\left\{\mu_{i} \mid i=1,2, \ldots, n\right\}, \Delta \mu_{j}$ is part of $\Delta \mu_{j}$, or $\Delta \mu_{j}$ is called a certain subset of $\Delta \mu_{j}$, then set $A=\left\{\Delta \mu_{j} \mid 0<j \leq n\right\}$ is called a clear subset of $U$, referred to as clear set.

Point out the following points: (1) Element $\mu_{j}$ in the domain $\mathrm{U}$ is interpreted as a classic set, its subset is a part of it. When $\Delta \mu_{j}, \Delta \mu_{j}=\mu_{j}$, it is clearly integrated into the classic set, so it is its promotion. (2) Here, the clear set is defined by the classic set, leaving out the characteristic function. (3) Each $\mu_{j}$ can be partly belonging to and partly not belonging to the clarity set $\mathrm{A}$. This is the ambiguity of this and the other, indicating that the clarity set can be used to describe the ambiguity of this and the other.

Definition 4 Existing classic set of $\mathrm{n}$ finite elements $\mu_{1}, \mu_{2}, \ldots, \mu_{a_{n}}$, its subset $\Delta \mu_{a}=\left\{a_{1}, a_{2}, \ldots, a_{i_{k}}\right\}$, among them $a_{i} \in R(1,2, \ldots, n)$, the membership function of clear subset $A=\left\{\Delta \mu_{1}, \Delta \mu_{2}, \ldots, \Delta \mu_{a_{n}}\right\}$ of domain $U=\left\{\mu_{1}, \mu_{2}, \ldots, \mu_{a_{n}}\right\}$.

$$
A(x)=\left\{\begin{array}{l}
\frac{\left|\Delta \mu_{a_{1}}\right|}{\left|\mu_{a_{1}}\right|}, x=\mu_{a_{1}} \\
\frac{\left|\Delta \mu_{a_{2}}\right|}{\left|\mu_{a_{2}}\right|}, x=\mu_{a_{2}} \\
\frac{\left|\Delta \mu_{a_{n}}\right|}{\left|\mu_{a_{n}}\right|}, x=\mu_{a_{n}}
\end{array}\right.
$$

When $\mu_{1}, \mu_{2}, \ldots, \mu_{a_{n}}$ is replaced by $a_{1}, a_{2}, \ldots, a_{n}$ correspondingly, we get:

$$
A(x)=\left\{\begin{array}{l}
\frac{\left|\Delta \mu_{a_{1}}\right|}{\left|\mu_{a_{1}}\right|}, x=a_{1} \\
\frac{\left|\Delta \mu_{a_{2}}\right|}{\left|\mu_{a_{2}}\right|}, x=a_{2} \\
\frac{\left|\Delta \mu_{a_{n}}\right|}{\left|\mu_{a_{n}}\right|}, x=a_{n}
\end{array}\right.
$$

Can be regarded as the domain of $\left\{a_{1}, a_{2}, \ldots, a_{n}\right\} \subset R$, a function with a value of $\left\{\frac{\left|\Delta \mu_{a_{1}}\right|}{\left|\mu_{a_{1}}\right|}, \frac{\left|\Delta \mu_{a_{2}}\right|}{\left|\mu_{a_{2}}\right|}, \ldots, \frac{\left|\Delta \mu_{a_{n}}\right|}{\left|\mu_{a_{n}}\right|}\right\} \subset[0,1]$, this function is called a clear rational number of order $\mathrm{n}$. When $\mathrm{n}=1$, $A(x)=\left\{\begin{array}{c}A\left(x_{1}\right), x=x_{1} \\ 0, x \notin\left\{x_{1}\right\}, \text { and } x \in R\end{array}\right.$ is a first-order clear rational number. In particular, when $A(x)=\left\{\begin{array}{c}A\left(x_{1}\right)=1, x=x_{1} \\ 0, x \notin\left\{x_{1}\right\}, \text { and } x \in R\end{array}\right.$, clear rational number $1 A(x)$ is represented by real number $x_{1}$, thus we can see that clear numbers are extensions of real numbers, and real numbers are special cases of clear numbers.

\subsubsection{Addition of clear rational numbers}

Definition 5 set clear rational numbers

$$
\begin{aligned}
& A(x)=\left\{\begin{array}{l}
A\left(x_{1}\right), x=x_{1}, \\
A\left(x_{2}\right), x=x_{2}, \\
\ldots \ldots \\
A\left(x_{n}\right), x=x_{n}, \\
0, x \in\left\{x_{1}, x_{2}, \ldots, x_{n}\right\}, \text { and } x \in R
\end{array}\right. \\
& B(x)=\left\{\begin{array}{l}
B\left(y_{1}\right), x=y_{1}, \\
B\left(y_{2}\right), x=y_{2}, \\
\ldots \ldots . \\
B\left(y_{n}\right), x=y_{m}, \\
0, x \in\left\{y_{1}, y_{2}, \ldots, y_{m}\right\}, \text { and } x \in R
\end{array}\right.
\end{aligned}
$$

Table 1 is called the edge and matrix of the possible values of $A(x)$ and $B(x)$. The real number columns $x_{1}, x_{2}, \ldots, x_{n}$ and $y_{1}, y_{2}, \ldots, y_{m}$ become the possible value sequences of $A(x)$ and $B(x)$, respectively. They are called the vertical and horizontal sides of the band edge and the matrix, and the two perpendicular lines are called 
the vertical and horizontal axes of the band edge and the matrix.

Table1. The possible values of $A(x)$ and $B(x)$ with edge and matrix

\begin{tabular}{ccccccc}
$x_{1}$ & $x_{1}+y_{1}$ & $x_{1}+y_{2}$ & $\cdots$ & $x_{1}+y_{j}$ & $\cdots$ & $x_{1}+y_{m}$ \\
\hline$\cdots$ & $\cdots$ & $\cdots$ & $\cdots$ & $\cdots$ & $\cdots$ & $\cdots$ \\
\hline$x_{i}$ & $x_{i}+y_{1}$ & $x_{i}+y_{2}$ & $\cdots$ & $x_{i}+y_{j}$ & $\cdots$ & $x_{i}+y_{m}$ \\
\hline$\cdots$ & $\cdots$ & $\cdots$ & $\cdots$ & $\cdots$ & $\cdots$ & $\cdots$ \\
\hline$x_{n}$ & $x_{n}+y_{1}$ & $x_{n}+y_{2}$ & $\cdots$ & $x_{n}+y_{j}$ & $\cdots$ & $x_{n}+y_{m}$ \\
\hline+ & $y_{1}$ & $y_{2}$ & $\cdots$ & $y_{j}$ & $\cdots$ & $y_{m}$ \\
\hline
\end{tabular}

Definition 6 Table 2 is called the marginal product matrix of membership of $A(x)$ and $B(x), A\left(x_{1}\right)$, $A\left(x_{2}\right), \ldots, A\left(x_{n}\right)$ and $B\left(y_{1}\right), B\left(y_{2}\right), \ldots, B\left(y_{m}\right)$ are called the membership sequence of $A(x)$ and $B(x)$ respectively, and they are called the vertical and horizontal sides of the marginal product matrix of membership. The two perpendicular lines are called the vertical and horizontal axes of the marginal product matrix.

Table2. Boundary product matrix of membership of $A(x)$ and $B(x)$

\begin{tabular}{ccccccc}
\hline$A\left(x_{1}\right)$ & $A\left(x_{1}\right) B\left(y_{1}\right)$ & $A\left(x_{1}\right) B\left(y_{2}\right)$ & $\cdots$ & $A\left(x_{1}\right) B\left(y_{j}\right)$ & $\cdots$ & $B\left(y_{m}\right)$ \\
\hline$\vdots$ & $\vdots$ & $\vdots$ & $\cdots$ & $\vdots$ & $\cdots$ & $\vdots$ \\
\hline$A\left(x_{i}\right)$ & $A\left(x_{i}\right) B\left(y_{1}\right)$ & $A\left(x_{i}\right) B\left(y_{2}\right)$ & $\ldots$ & $A\left(x_{i}\right) B\left(y_{j}\right)$ & $\cdots$ & $A\left(x_{i}\right) B\left(y_{m}\right)$ \\
\hline$\vdots$ & $\vdots$ & $\vdots$ & $\cdots$ & $\vdots$ & $\cdots$ & $\vdots$ \\
\hline$A\left(x_{n}\right)$ & $A\left(x_{n}\right) B\left(y_{1}\right)$ & $A\left(x_{n}\right) B\left(y_{2}\right)$ & $\cdots$ & $A\left(x_{n}\right) B\left(y_{j}\right)$ & $\cdots$ & $A\left(x_{n}\right) B\left(y_{m}\right)$ \\
\hline$\times$ & $B\left(y_{1}\right)$ & $B\left(y_{2}\right)$ & $\cdots$ & $B\left(y_{j}\right)$ & $\cdots$ & $B\left(y_{m}\right)$
\end{tabular}

Definition 7: A matrix composed of the possible values of $A(x)$ and $B(x)$ with edges and the upper right number in the matrix

$$
\left[\begin{array}{cccc}
a_{11} & a_{12} & \cdots & a_{1 m} \\
\vdots & \vdots & & \vdots \\
a_{i 1} & a_{i 2} & \cdots & a_{i m} \\
\vdots & \vdots & & \vdots \\
a_{n 1} & a_{n 2} & \cdots & a_{n m}
\end{array}\right]
$$

Called $A(x)$ and $B(x)$ possible values and matrix.

Definition 8 the matrix composed of the upper right numbers in the marginal product matrix of membership of $A(x)$ and $B(x)$

$$
\left[\begin{array}{cccc}
b_{11} & b_{12} & \cdots & b_{1 m} \\
\vdots & \vdots & & \vdots \\
b_{i 1} & b_{i 2} & \cdots & b_{i m} \\
\vdots & \vdots & & \vdots \\
b_{n 1} & b_{n 2} & \cdots & b_{n m}
\end{array}\right]
$$

It is called the membership product matrix of $A(x)$ and $B(x)$.

Definition 9 the possible values of $A(x)$ and $B(x)$ and the product of element $a_{i j}$ in the i-th row and $\mathrm{j}$-th column of the matrix and their membership degree matrix $b_{i j}$ are called corresponding elements.
Definition 10: Arrange the possible values of $A(x)$ and $B(x)$ and the elements in the matrix into a column $x_{1}, x_{2}, \ldots, x_{l}$, and arrange the corresponding elements of $x_{i}(i=1,2, \ldots, l)$ in the membership product matrix of $A(x)$ and $B(x)$ into a column $C\left(x_{1}\right), C\left(x_{2}\right), \ldots, C\left(x_{l}\right)$, . This is called a clear number.

$$
C(x)=\left\{\begin{array}{l}
C\left(x_{1}\right), x=x_{1}, \\
C\left(x_{2}\right), x=x_{2}, \\
\cdots \\
C\left(x_{l}\right), x=x_{l}, \\
0, x \in\left\{x_{1}, x_{2}, \ldots, x_{l}\right\}, x \in R
\end{array}\right.
$$

Is the sum of $A(x)$ and $B(x)$, denoted as $C(x)=A(x)+B(x)$.

\subsubsection{Multiplication of clear rational numbers}

Definition 11 if the matrix in the above definition 8 is changed to a matrix composed of the upper right numbers in the marginal product matrix of $A(x)$ and $B(x)$, it is called the possible value product matrix of $A(x)$ and $B(x)$. Then arrange the elements in the matrix of possible value products of $A(x)$ and $B(x)$ into a column $x_{1}, x_{2}, \ldots, x_{l}$, Arrange the corresponding elements of 
$x_{i}(i=1,2, \ldots, l)$ in the membership product matrix of $A(x)$ and $B(x)$ into a column of $C\left(x_{1}\right), C\left(x_{2}\right), \ldots, C\left(x_{l}\right)$, which is called the clear number

$$
C(x)=\left\{\begin{array}{l}
C\left(x_{1}\right), x=x_{1}, \\
C\left(x_{2}\right), x=x_{2}, \\
\ldots \\
C\left(x_{l}\right), x=x_{l}, \\
0, x \in\left\{x_{1}, x_{2}, \ldots, x_{l}\right\}, x \in R
\end{array}\right.
$$

Is the product of $A(x)$ and $B(x)$, denoted as $C(x)=A(x) \times B(x)$.

\subsubsection{Mean value of clear rational numbers}

Definition 12 let $A(x)$ be a clear rational number of order $\mathrm{n}$, which can be expressed as

$$
A(x)=\left\{\begin{array}{l}
A\left(x_{1}\right), x=x_{1}, \\
A\left(x_{2}\right), x=x_{2}, \\
\cdots \ldots . \\
A\left(x_{n}\right), x=x_{n}, \\
0, x \in\left\{x_{1}, x_{2}, \ldots, x_{n}\right\}, \text { and } \quad x \in R
\end{array}\right.
$$

Then the mean value of the clear rational number $A(x)$ is:

$$
E(A(x))=\frac{\sum_{i=1}^{n} x_{i} A\left(x_{i}\right)}{\sum_{i=1}^{n} A\left(x_{i}\right)}
$$

\section{Optimal model of experimental teaching program with clear theory improvement}

Step 1 initially determine the alternative experimental teaching plan.

Step 2 Form an evaluation team and determine the evaluation attribute set and scale set.

Step 3 Ask the evaluation team to make a fuzzy evaluation of each alternative, and obtain the comprehensive evaluation value of the alternative given by the group $d_{k} \cdot z_{i}^{(k)}(\mathrm{i}=1,2, \ldots, \mathrm{n} ; \mathrm{k}=1,2, \ldots, \mathrm{p})$ $z_{i}^{(k)}=\sum_{j=1}^{m} \lambda_{j} x_{i j}$ Where $\lambda_{j}$ is the weighting factor, $\sum_{j=1}^{m} \lambda_{j}=1$, thus, the comprehensive evaluation matrix $\mathrm{P}$ of option $p_{i}$ given by group $d_{k}$ can be obtained.

Step 4 Establish a team of experts, give feedback on the evaluation results, and the expert group will make statements on the evaluation results, and use clear theory to calculate the statement results, and solve the comprehensive results after the feedback of each plan $E\left(P_{i}(x)\right)$.

Step 5 Determine the pros and cons of each alternative pivot plan.

The larger the value of $E\left(P_{i}(x)\right)$, the better the comprehensive value of the evaluation and feedback results, and the better the corresponding plan

\section{Conclusion}

This paper combines the triangular fuzzy number with the clarity theory and innovatively proposes an improved experimental teaching program optimization model of the clarity theory, which provides a new method for the evaluation of the experimental teaching program. The order of the mean size of the comprehensive decision value after feedback can be seen that the ranking of the pros and cons of the alternatives.

\section{References}

1. Xu Yin, Zhao Jiao, Lei Cuiyu, et al. Necessity and countermeasures of experimental teaching reform in local colleges and universities under the background of "double first-class" construction[J]. High Education Journal 2019 (18): 138-140.

2. Yu Songkun, Xi Jie. Deepen the reform of practical teaching and cultivate students' innovative ability [J]. China University Teaching, 2009 (8): 68-70.

3. Guan Lihe, Lu Hao, Jiang Wei, et al. Experimental teaching reform and innovative talent training[J]. Laboratory Research and Exploration, 2011 (7):278$280+307$.

4. Wu Huaying, Wu Heqin. Clarity set and its application [M]. Hong Kong: Hong Kong News Publishing House, 2007.

5. Su Fahui. A clear theoretical basis [M]. Hefei University of Technology Press, 2013.

6. $\mathrm{Xu}$ Xingshuang, Liu Lantian. The application of fuzzy analytic hierarchy process in selecting the best part-time job plan [J]. Think Tank Times, 2019 (39): 187-188.

7. Deng Dexue, Xu Xinrui. Research on engineering bid evaluation method based on fuzzy decision theory [J]. Shanxi Architecture, 2019, 45(10): 218-219.

8. Fu Xu, Liu Yiliang, Liu Xiaoli. Research on grayfuzzy multi-attribute group decision-making model of soft foundation treatment scheme based on analytic hierarchy process $[\mathrm{J}]$. Journal of Beihua Institute of Aeronautics and Astronautics, 2017, 27(04): 1-4.

9. Reda B, Didier C. A decision-making computational methodology for a class of type-2 fuzzy intervals: An interval-based approach [J]. Information Sciences, 2019(2), 510: 256-282.

10. Ma J, Ji C. Generalized Grey Target Decision Method for Mixed Attributes Based on Connection Number [J]. JOURNAL OF APPLIED MATHEMATICS, 2014: 1-8.

11. Ye J. Fuzzy decision-making method based on the weighted correlation coefficient under intuitionistic fuzzy environment [J]. European Journal of Operational Research, 2010, 205(1): 202-204.

12. Fu Xiaoquan. Application of Fuzzy Comprehensive Evaluation in Teaching Quality Evaluation [J]. Contemporary Education Forum, 2005(3): 37-40. 
13. Pei Z, Zheng L. A novel approach to multi-attribute decision making based on intuitionistic fuzzy sets [J]. Expert Systems with Applications, 2012, 39(3): 25602566.

14. Su Fahui. Two mistakes of fuzzy sets [J]. Journal of Jishou University (Natural Science Edition), 2012, 33(2): 13-15.

15. Wu Heqin, Ji Hongyan. Fuzzy topology is wrong. Journal of Hebei University of Engineering, 2008, 25(1): 111-112.

16. Wu Heqin, Wu Huaying, Su Yu. A wrong theorem introduced by fuzzy set theory [J]. Journal of Hebei Institute of Architecture and Technology, 2006, 23(1): 108-109.

17. Wang Weiming, Wang Guofu, Feng Yuguo. Triangular fuzzy number multi-attribute decisionmaking grey optimization model and its application in the evaluation of engineering design schemes [J]. Mathematics in Practice and Knowledge, 2012, 42(12): 21-27.

18. Liu Xiumei, Zhao Keqin, Wang Chuanbin. A New Triangular Fuzzy Number Multi-Attribute Decision Model Based on Connection Number [J]. Systems Engineering and Electronic Technology, 2009, 31(10): 2399-2403. 